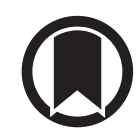

CrossMark

\title{
Nonadherence with inhaled preventer therapy in severe asthmatic patients on long-term omalizumab
}

\author{
To the Editor:
}

We read with interest the paper by LEE et al. [1], reporting on the high rate of nonadherence in a group of severe asthma patients who were potentially suitable for biological therapy or bronchial thermoplasty. In our experience the issue of nonadherence is not confined to patients under active consideration for biological therapy. We have identified significant rates of nonadherence in a group of patients on long-term Omalizumab therapy.

In the Northwest of England, patients eligible for biological therapy are reviewed by the regional multidisciplinary team (MDT) meeting and only patients classed as adherent to their prescribed preventer therapy, as measured by medication pick up rates from the patients' General Practitioner (GP) of $80 \%$ or higher, are approved for therapy. Therefore, at the time of initiation of therapy these patients were classed as adherent.

We have looked at the medication pick up rates for 79 severe asthma patients attending for Omalizumab therapy in our centre. Over a 2-week period patients attending for routine Omalizumab care (post 16 week assessment) underwent standard observations, namely: forced expiratory volume in $1 \mathrm{~s}$ (FEV1), exhaled nitric oxide fraction $(\mathrm{FeNO})$ and Asthma Control Questionnaire 7 (ACQ-7). Additionally, each patient was asked to clarify the type and frequency of current inhaled corticosteroid (ICS) or ICS/long-acting $\beta$-agonist (LABA) combination inhaler used and adherence data was obtained from the patients GP for the last 6 months. Nonadherence was defined as failure to pick up $80 \%$ of the prescribed dose of preventer therapy over the previous 6 months.

ICS/LABA or ICS adherence was observed in 39 patients (49.4\%). Comparison between the two groups is shown in table 1 . There was little difference between the groups in any of the recorded parameters. Both groups demonstrated a significant improvement in ACQ-7 from baseline and there was a trend $(\mathrm{p}=0.067)$ for better control (lower ACQ-7 score) in the nonadherent group. The FeNO result was higher in the nonadherent group, but in both groups FeNO was within the normal range $(<25 \mathrm{ppb})$ and was not thought to be of any clinical significance. The prescribed dose of inhaled steroid was higher in the adherent group.

The rate of nonadherence was $50.6 \%$, which was startling and was of a similar magnitude to that seen in other published studies in severe asthma $[2,3]$. Looking at the nonadherent group, 26 patients $(78.8 \%)$ were picking up less than $50 \%$ of their inhaled preventer therapy, indicating a dramatic fall in adherence amongst patients on long-term Omalizumab who had demonstrated a significant response to treatment as measured by improvements in asthma control and quality of life. The nonadherent patients did not show any signs of deterioration in asthma control or any clinically meaningful changes in measures of airway inflammation $(\mathrm{FeNO})$. In fact the trend to better self-reported control, as measured by the ACQ-7, has led us to postulate that if patients perceive themselves to be well controlled they are more inclined to drop off their inhaled preventer therapy. It has been reported elsewhere that patients are likely to become nonadherent if they no longer perceive benefit or need from the treatment that they are taking [4].

What is not known is the difference in exacerbation rates between the adherent and nonadherent patients and this is subject to further evaluation. The concern is that nonadherent patients would be at increased

@ERSpublications

Nonadherence with inhaled preventer therapy is prevalent in previously adherent patients on longterm omalizumab http://ow.ly/5wdE30kp0MM

Cite this article as: Allen DJ, Holmes L-J, Hince KA, et al. Nonadherence with inhaled preventer therapy in severe asthmatic patients on long-term omalizumab. Eur Respir J 2018; 52: 1801025 [https://doi.org/ $10.1183 / 13993003.01025-2018]$. 
TABLE 1 Comparison of adherent and nonadherent groups

\begin{tabular}{lcc} 
Parameter & Adherent group & Nonadherent group \\
\hline Duration of treatment months & 32 & $33(p=0.55)$ \\
Baseline FEV1 \% predicted & 55.0 & $61.95(p=0.26)$ \\
Current FEV $\%$ predicted & 65.71 & $69.85(p=0.39)$ \\
Baseline ACQ-7 score & 3.85 & $2.85(p=0.40)$ \\
Current ACQ-7 score & 2.36 & $1.79(p=0.067)$ \\
BDP equivalent ICS dose $\boldsymbol{\mu g}$ & 2000 & $1600(p=0.04)$ \\
Current FeNo ppb & 15 & $22(p=0.048)$ \\
Blood eosinophil count $\times \mathbf{1 0}^{\mathbf{9}}$ per L & 0.08 & $0.18(p=0.09)$
\end{tabular}

FEV1: forced expiratory volume in $1 \mathrm{~s}$; ACQ-7: Asthma Control Questionnaire 7; BDP: beclometasone; ICS: inhaled corticosteroid; FeNO: exhaled nitric oxide fraction.

risk of an asthma exacerbation, although anecdotally we have not seen many exacerbations in patients on long-term Omalizumab.

What is clear from our findings is that adherence with therapy cannot be taken for granted in patients on Omalizumab and that from clinical parameters alone it is not possible to identify nonadherence. These are patients that are all seen in our unit every 2 or 4 weeks depending on dosing schedule and, despite regular contact with our specialist nurses, nonadherence went undetected. Going forward it is clear that systems need to be put in place to regularly monitor medication pick up rates for preventer therapy in severe asthma patients receiving biological therapy. For our patients approved for Mepolizumab, all patients sign an undertaking at the start of therapy that stipulates continued adherence with preventer therapy in order to continue to receive therapy. There is currently no such agreement for the Omalizumab patients and we will be looking at the impact on adherence of this treatment contract in a further study.

Our finding also poses the question as to what to do with patients on biological therapy who remain nonadherent with their inhaled preventer therapy. If patients are not able to return to previous levels of adherence, is it safe to withdraw biological therapy? Certainly what is effectively mono-therapy with Omalizumab is expensive, but if a severe asthma exacerbation occurs leading to hospitalisation, as well as intensive care admission or even death, this cost comparison is less clear cut in purely financial terms.

Our findings show that adherence cannot be taken for granted and that adherent severe asthma patients on long-term Omalizumab become nonadherent without any apparent detriment in asthma control. The severe asthma community needs to decide if it is acceptable to provide expensive biological therapies to nonadherent patients who are unable to improve or maintain adherence with inhaled preventer therapy.

David J. Allen, Leanne-Jo Holmes, Kerry A. Hince, Rachael Daly, Calra Ustabashi and Gael Tavernier Severe Asthma Service, Northwest Lung Centre, Wythenshawe Hospital, Manchester, UK.

Correspondence: D.J. Allen, Severe Asthma Service, Northwest Lung Centre, Wythenshawe Hospital, Manchester, M23 9LT, UK. E-mail: david.allen@mft.nhs.uk

Received: May 312018 | Accepted: June 042018

Conflict of interest: D.J. Allen reports non-financial support from Mundipharma for attendance at the ERS meeting, and speaker fees for presenting at an educational meeting sponsored by Astra Zeneca, outside the submitted work. L-J. Holmes reports personal fees for speaking at conferences from Novartis and TEVA, outside the submitted work.

\section{References}

1 Lee J, Tay TR, Radhakrishna N, et al. Nonadherence in the era of severe asthma biologics and thermoplasty. Eur Respir J 2018; 51: 1701836.

2 Gamble J, Stevenson M, McClean E, et al. The prevalence of nonadherence in difficult asthma. Am J Respir Crit Care Med 2009; 180: 817-822.

3 Murphy AC, Proeschal A, Brightling CE, et al. The relationship between clinical outcomes and medication adherence in difficult-to-control asthma. Thorax 2012; 67: 751-753.

4 Horne R, Chapman SC, Parham R, et al. Understanding patients' adherence-related beliefs about medicines prescribed for long term conditions: a meta-analytic review of the Necessity-Concerns Framework. PLoS One 2013; 8: e80633. 